\title{
Eine kleine Revue literaturtheoretischer Logiken des Fragments
}

\section{Eine vernachlässigte Relation: Fragment und Literaturtheorie}

Die Frage nach der Produktivität des literaturwissenschaftlichen Umgangs mit Fragmenten, Entwürfen, Notizen sowie allen anderen Formen des Offenen stellt der vorliegende Band zum einen bewusst weit, was den Fokus Theodor Fontane angeht, zum anderen aber zugleich auch sehr konkret. Einen gemeinsamen Fluchtpunkt können dieser spezifische Bezugspunkt Fontane und das breite Spektrum der ,Formen des Offenen' dann gewinnen, wenn man sie mit demjenigen Nachdenken über unser wissenschaftliches Handeln konfrontiert, das als literaturwissenschaftliche Theoriebildung auf einer Metaebene erfolgt. ${ }^{1}$

Genau das soll im Folgenden versucht werden, nämlich die Formen des Offenen und einige ausgewählte literaturtheoretische Positionen aufeinander zu beziehen und danach zu fragen, welcher Status Fragmenten, Entwürfen, Notizen und anderen Formen des Offenen aus Sicht der verschiedenen Literaturtheorien eigentlich zukommt. In dieser Perspektive geht es nicht mehr darum, von unten nach oben zu bestimmen, was genau ein Fragment, was ein Entwurf, was eine Skizze ist, nicht darum, eine vorgängige Vorstellung oder gar Definition von Fragment mit diversen Theorieentwürfen zu konfrontieren (das wäre auch kaum möglich, steht eine Gattungstheorie des Fragments und im Weiteren eine aller Formen des Offenen doch noch weitgehend aus). ${ }^{2}$ Vielmehr geht es darum, die Perspektive umzukehren, die Logiken des Fragments aus Sicht der verschiedenen Literaturtheorien in den Blick zu nehmen und aufzuzeigen, wie diese das Fragmentarische jeweils spezifisch konstituieren: Wie denkt die Hermeneutik das Fragment? Was kann die Dekonstruktion mit Entwürfen anfangen? Warum hat es der literaturwissenschaftliche Positivismus mit Notizen vergleichsweise leicht, und zwar ebenso wie die Diskursanalyse? Wen oder was legt eigentlich eine litera-

1 Für wichtige Hinweise und Anregungen danke ich Iuditha Balint, Britta Caspers und Maren Jäger.

2 Eine Ausnahme bildet Justus Fetscher, Bruchstückwerke. Stationen einer Ästhetik des Fragments (1790-1970). Habilitationsschrift am Fachbereich Philosophie und Geisteswissenschaften der Freien Universität Berlin 2008. 
turpsychologische Analyse mit einem unvollendet gebliebenen Briefentwurf auf die berühmte Couch? Und wie (wenn überhaupt) konzipieren die verschiedenen Theoriemodelle jeweils das Pendant der ,Formen des Offenen', nämlich die für abgeschlossen geltenden Einzelwerke, von denen nicht alle Literaturtheorien (und auch nicht alle Autoren ${ }^{3}$ ) mit dieser Emphase sprechen mögen?

Dieses Unterfangen der Korrelation von Formen des Offenen mit literaturtheoretischen Positionen scheint insofern sinnvoll $\mathrm{zu}$ sein, als es geeignet ist, die Prämissen und Postulate des eigenen Denkens zu hinterfragen, das heißt Theoriebildung zum Nachdenken über das eigene Handeln und Vorgehen zu nutzen, auch dann, wenn einem dabei vielleicht einmal der vermeintlich feste Boden des eigenen Forschens entzogen wird. Für Jonathan Culler besteht daher der Sinn aller Theoriebildung darin, „durch eine Befragung von Prämissen und Postulaten genau das zu zerstören, von dem man geglaubt hat, es zu wissen“. ${ }^{4}$ Das mag sich im ersten Moment nun ziemlich destruktiv anhören, schließt Bestätigung aber nicht aus, so dass Culler etwas abgemildert werden kann: Der Bezug von literaturwissenschaftlicher Arbeit an Fragmenten und anderen literarischen Formen des Offenen auf Literaturtheorien kann uns vielleicht die eine oder andere Voraussetzung, Prämisse und Bedingung unseres Bemühens deutlicher machen, als sie uns in der praktischen literaturwissenschaftlichen Arbeit vor Augen steht. Kurz: Der theoretische Kontroll-Blick kann einen vor Irrtümern bewahren.

Die Komplexität der Bestimmung einer ,Logik des Fragments‘ liegt aber nicht nur auf Seiten der Literaturtheorien, sondern auch auf derjenigen der Vielfalt der Formen des Offenen. Denn allein schon das, was jeweils unter ,Fragment‘ verstanden wird, kann sehr stark divergieren, so dass eigentlich eine ganze Liste der Formen des Offenen und ihrer jeweiligen ästhetisch-philosophischen Konzeptualisierungen einer nicht weniger komplexen Liste an literaturtheoretischen Ansätzen gegenübergestellt werden müsste. Bei der Rede von ,Entwurf‘ beispielsweise hätte man zumindest zwischen später zum Werk ausgearbeitetem Entwurf und solchen Formen des Offenen zu unterscheiden, die Entwurf geblieben sind. Noch komplexer sieht es für die Bezeichnung ,Fragment‘ aus, die gleich mehrfach besetzt ist, unter anderem überlieferungstechnisch, editionsphilologisch, poetologisch und programmatisch-ästhetisch, weshalb hier ein wenig davor zurückgeschreckt wird, von Begriff zu sprechen. So referiert die Bezeichnung ,Fragment`

3 Vgl. mit Blick auf Friedrich Schiller dazu Dirk Oschmann, Die Aporien des ,Ganzen‘. In: „Ein Aggregat von Bruchstücken“. Fragment und Fragmentarismus im Werk Friedrich Schillers, hg. von Jörg Robert unter Mitarbeit von Marisa Irawan, Würzburg 2013, S. 249-267.

4 Jonathan Culler, Literaturtheorie. Eine kurze Einführung, Stuttgart 2002 (Reclams Univ.-Bibl. 1866), S. 30. 
auf zumindest zwei einander diametral entgegenstehende Sachverhalte, nämlich zum einen auf losgelöste Teile von vormals vollständigen Kunstwerken, die nicht als Ganze überliefert sind (solche beschreibt Fontane für plastische Kunstwerke, aber auch essayistische Texte gelegentlich in den Wanderungen durch die Mark Brandenburg; ${ }^{5}$ in der Literaturwissenschaft spielen sie vor allem in der Mediävistik eine Rolle), zum anderen aber auch auf solche Teile, Anfänge, Bruchstücke oder Vorstufen von Kunstwerken, die nie Ganzheits- und damit Werkcharakter erreicht haben. ${ }^{6}$ Diese Vieldeutigkeit der Bezeichnung ,Fragment' bleibt selbst dann bestehen, wenn man sich nur auf den Aspekt des Unvollendeten, des Fragments im Sinne von Entwurf und Skizze konzentriert, der - diesmal anders als in der Mediävistik - in der neueren deutschen Literaturwissenschaft das vielleicht gängigste Verständnis von Fragment darstellt. ${ }^{7}$

Noch einmal komplizierter wird es schließlich, wenn das Kunstwerk in seiner Ganzheit von Beginn an als Fragment intendiert ist, wenn das Fragment - wie es Klamer Eberhard Karl Schmidt 1790 in seinen Neuen poetischen Briefen so schön

5 Vgl. Theodor Fontane, Wanderungen durch die Mark Brandenburg. Dritter Teil. Havelland. Die Landschaft um Spandau Potsdam, Brandenburg, hg. von Gotthard Erler und Rudolf Mingau, 2. Aufl., Berlin 1994 (GBA), S. 169: „Das Fragment einer antiken Sarkophagskulptur, welche den Raub der Proserpina darstellt“, sowie Theodor Fontane, Wanderungen durch die Mark Brandenburg. Zweiter Teil. Das Oberland. Barnim-Lebus, hg. von Gotthard Erler und Rudolf Mingau, 2. Aufl., Berlin 1994, (Große Brandenburger Ausgabe) S. 108: „Thaer selbst schreibt über diese später in etwas veränderter Gestalt so berühmt gewordene Arbeit: ,Ich erschuf mir damals [...] ein selbständiges, religionsphilosophisches System und brachte es flüchtig zu Papier. Es ward wider meinen Willen abgeschrieben, fiel in die Hände eines großen Mannes, der den Stil etwas umänderte und einen Teil davon, als Fragment eines unbekannten Verfassers, herausgab. Bis jetzt wissen es nur drei lebende Menschen, daß ich der Urheber bin.' In diesen Worten Thaers wird weder Lessing genannt noch mit Bestimmtheit angegeben, welches der ,Fragmente eines Wolfenbüttelschen Unbekannten` Thaer für sich in Anspruch nimmt [...].“

6 Vgl. Peter Strohschneider, Fragment ${ }_{2}$. In: Reallexikon der deutschen Literaturwissenschaft. Neubearb. des Reallexikons der deutschen Literaturgeschichte, gemeinsam mit Harald Fricke, Klaus Grubmüller und Jan-Dirk Müller hg. von Klaus Weimar, Bd. I, Berlin, New York 1997, S. 624 f., hier 624. - Ernst Behler, Das Fragment. In: Prosakunst ohne Erzählen. Die Gattungen der nicht-fiktionalen Kunstprosa, hg. von Klaus Weissenberger, Tübingen 1985, S. 125-143, hier $125 \mathrm{f}$.

7 Sinnvoll wäre zudem eine Abstufung der ,Formen des Unvollendeten' nach dem Grad der Annäherung an abgeschlossene Werke, etwa in der Reihenfolge Skizze, Entwurf, Fragment, doch scheint der bisherige Sprachgebrauch hier eher uneinheitlich zu sein. Eine noch einmal andere Form der Fragmentarität wäre die Segmentierung eines vormals vollständigen oder als zu vervollständigen intendierten Textes, etwa bei Zeitschriftenvorabdrucken von Romanen in Tageszeitungen und Rundschauzeitschriften. 
formuliert hat - „unergänzlich“ ist. ${ }^{8}$ Das ist beispielsweise dann gegeben, wenn die fragmentarische Überlieferung griechischer Statuen in späterer Zeit zum ästhetischen Ideal erhoben wird und moderne Bildhauer wie etwa Auguste Rodin Torsi, wenn Architekten Ruinen oder wenn Literaten wie die der Romantik von vornherein unvollendete, als Fragmente intendierte Texte schaffen, ${ }^{9}$ die einer „Ästhetik des Skizzenhaften“10 folgen, wie sie vom frühen Friedrich Schlegel über Friedrich von Hardenberg bis hin zu Ludwig Tieck in vielfältiger Weise anzutreffen ist. ${ }^{11}$ Darauf wiederum wird bis heute Bezug genommen, beispielsweise mit Christian Geisslers Roman Kamalatta von 1988, der sich im Untertitel als Romantisches Fragment annonciert. Hier haben wir den Fall, dass der Text als Roman durchaus Vollständigkeit signifiziert, diese mit dem Untertitel aber zugleich zurücknimmt, relativiert und an die Poetologie der Romantik zurückbindet. ${ }^{12}$

Allein schon aufgrund solcher Ambivalenzen, die deutlich machen, dass „Fragment und Totalität [...] korrelativ“ zu denken sind, und „über das eine [...] nicht zu sprechen“ ist „ohne das andere“, ${ }^{13}$ und sei es in Form seiner Abwesenheit, ist der Status und Stellenwert, den Fragmente in den verschiedenen Literaturtheorien besitzen, höchst verschieden. ${ }^{14}$ Dies zumal, da Literaturtheorien mal stärker den Vorgang der literarischen Produktion (und mit ihm auch Fragmente aus Sicht der Produzenten), mal stärker den Vorgang der Rezeption (und mit ihm

8 Klamer Eberhard Karl Schmidt, Neue poetische Briefe, Braunschweig 1790, Brief 116; zit. nach: Deutsches Wörterbuch von Jacob und Wilhelm Grimm, 16 Bde. in 32 Teilbden, Leipzig 1854-1961, Bd. 4, Sp. 55, http://woerterbuchnetz.de/DWB/?sigle=DWB\&mode=Vernetzung\&hitlist=\&pattern list=\&lemid=GF07915\#XGF07915 (7. 10. 2016).

9 Vgl. Justus Fetscher, Fragment. In: Ästhetische Grundbegriffe. Ein historisches Wörterbuch in 7 Bden., hg. von Karlheinz Barck, Martin Fontius, Friedrich Wolfzettel und Burkhart Steinwachs, Bd. 2, Stuttgart, Weimar 2001, S. 551-588, hier $573 \mathrm{f}$.

10 George Steiner, Das totale Fragment. In: Fragment und Totalität, hg. von Lucien Dällenbach und Christiaan L. Hart Nibbrig, Frankfurt am Main 1984 (edition suhrkamp 1107), S. 18-29, hier 19. 11 Vgl. Fetscher, Fragment (wie Anm. 9), S. 560-570. - Rüdiger Bubner, Gedanken über das Fragment. Anaximander, Schlegel und die Moderne. In: Merkur. Zeitschrift für europäisches Denken, 47, 4, 1993, S. 290-299. - Dirk Schröder, Fragmentpoetologie im 18. Jahrhundert und bei Friedrich von Hardenberg. Untersuchungen zur vergleichenden Rekonstruktion der impliziten Poetologie von Aphorismus und Fragment im ausgehenden 18. Jahrhundert, Diss. Kiel 1976.

12 Christian Geissler, Kamalatta. Romantisches Fragment, Berlin 1988.

13 Lucien Dällenbach/Christiaan L. Hart Nibbrig, Fragmentarisches Vorwort. In: Fragment und Totalität, hg. von L. Dällenbach und Ch. L. Hart Nibbrig, Frankfurt am Main 1984 (edition suhrkamp 1107), S. 7-17, hier 7.

14 Vgl. die von Michael Braun unterschiedenen „Formen des Fragments“, nämlich „[ü]berlieferungsbedingte Fragmente“, „[p]roduktionsbedingte Fragmente“ und „[k]onzeptionelle Fragmente“ (M. Braun, „Hörreste, Sehreste.“ Das literarische Fragment bei Büchner, Kafka, Celan und Benn, Köln, Weimar, Wien 2002, S. 16-24). 
Fragmente aus Sicht der Rezeption, der Leser), ${ }^{15}$ mal stärker die literarischen Texturen selbst in den Vordergrund rücken (und damit die literarischen Strukturen und Verfahren von Fragmenten, auch in Bezug auf und in Vergleich zu anderen unabgeschlossenen wie abgeschlossenen Texten). Alles dies kann wiederum in zweierlei Hinsicht geschehen, nämlich zum einen, um die Produktionsprozesse ,auf dem Weg zum Werk' nachzuzeichnen (eventuell über mehrere Stationen und unterschiedliche Modi von Fragmenten hinweg), so dass die Fragmente als Unterbau der späteren Werke erscheinen, und zum anderen, um Fragmente wie auch abgeschlossene Texte besser verstehen zu können.

Noch einmal eine andere Ebene bildet schließlich die jeweilige mediale Verfasstheit, die mitunter auch abgeschlossene Texte als Fragmente erscheinen lässt, so beispielsweise dann, wenn sie bei der seriellen Publikation in Zeitschriften re-fragmentiert werden. Die mediale Rahmenbedingung der Serialität führt dann zunächst zur Segmentierung, die - wenn man sie als Fragmentierung auffasst auch das einzelne Segment als Fragment erscheinen lässt. Allerdings bliebe hier zu berücksichtigen, dass Texte - gerade in der zweiten Hälfte des 19. Jahrhunderts - vielfach in die Serialität der Zeitschriftenvorabdrucke förmlich hineingeschrieben werden, so dass die daraus resultierende Fragmentierung bereits Teil des Werkganzen wäre.

\section{Literaturtheoretische Logiken des Fragments}

Dem Status des Fragments soll daher im Folgenden für einige literaturwissenschaftliche Theoriemodelle nachgegangen und nach den jeweiligen Logiken ihres theoretischen Blicks auf Fragmente (im Sinne von Skizzen, Entwürfen und Vorstufen) gefragt werden.

\section{II.1 Positivismus, Geistesgeschichte, Werkimmanenz, Formalismus}

Einer der ersten literaturwissenschaftlichen Theorieansätze ist der ab Mitte des 19. Jahrhunderts und bis in die 1910er Jahre wirkmächtige Positivismus, der - orientiert am Ideal der Naturwissenschaften - Fakten erheben, vorliegende Realien und Realitäten beobachten und sie in Beziehung zueinander setzen wollte. Dabei

15 M. Braun (wie Anm. 14), „Hörreste, Sehreste“, S. 25-29. 
ging es vor allem um kausale Relationen. Erhoben (weniger interpretatorisch erarbeitet) werden sollte ein positives Wissen über Autoren, über Texte und auch über die Beziehung beider zum Publikum; eine besondere Rolle spielte die Sicherung der philologisch für ,richtig` erachteten Texte durch kritische Editionen. Das Fragment wie auch alle anderen Spielarten des Unvollendeten waren dabei zunächst einmal Materialien unter anderen Materialien, ${ }^{16}$ die als Hilfsmittel vor allem für die Textedition, z.B. Datierungsfragen, herangezogen wurden, denen aber kaum ein eigener ästhetischer oder gar poetologischer Wert zukam.

Mit der Diachronisierung des Positivismus in Form von geschichtsphilosophisch, das heißt mal mehr mal weniger teleologisch angelegten Literaturgeschichten, die zugleich den Übergang zu den verschiedenen Formen von Geistesgeschichte bzw. Geistestypologie markieren, rückt das Interesse an Fragmenten in den Hintergrund, geht es doch jetzt in der einen Linie um das Zusammendenken literarischer und politischer ,Höhe-“ und ,Wendepunkte', in der anderen um die Darstellung ,geistiger Gesamtzusammenhänge‘, ihr allmähliches Sich-Durchsetzen und dann auch wieder ihre Ablösung durch neue Ideen. Dabei spielen - in deutlichem Gegensatz zur Hermeneutik - die Autor-Biographien keine so wichtige Rolle mehr; auch der Stellenwert der Textedition ist relativiert und mit beidem auch hier das Interesse an Fragmenten. Denn der theoretisch-methodische Fokus der geisteswissenschaftlichen Literaturwissenschaft richtet sich auf die Gestalt der Texte, die als äußere Objektivation eines inneren Geistes verstanden wird. Für den Nachweis der Entsprechung von Gestalt und Geist waren alle ,Formen des Offenen' aber denkbar ungeeignet. Über die Geistesgeschichte hinaus gilt das auch für das Denkmodell der Werkimmanenz (und eben nicht: Fragment-Immanenz oder Entwurfs-Immanenz), die hinsichtlich des Ausblendens von Kontextinformationen und des daraus folgenden mangelnden Interesses an Fragmenten und Ähnlichem auf erstaunliche Weise mit dem russischen Formalismus konform geht, der den literarischen Text zunächst einmal ebenfalls als einen autonomen Bereich betrachtet.

\section{II.2 Hermeneutik, Rezeptionsästhetik, Genieästhetik}

Ganz anders sieht es für die Formen des Offenen im Falle der literaturwissenschaftlichen Hermeneutik aus. Denn aus ihrer Perspektive ist das Fragment als Zugang zu Werk und Autor und damit als geradezu privilegiertes Element im her-

16 Vgl. dazu Rainer Baasner/Maria Zens, Methoden und Modelle der Literaturwissenschaft. Eine Einführung, 3. Aufl., Berlin 2005, S. 55 f. 
meneutischen Zirkel des Verstehens von Interesse (darin im Übrigen nicht unähnlich dem Autor-Brief). Dieser Zugang besteht in erster Linie darin, dass Fragmente eine geradezu ideale Ausgangssituation für das eröffnen, was die Hermeneutik ,Horizontverschmelzung، genannt hat, scheinen sie doch noch näher an der Autorinstanz zu liegen, mehr von ihr und dem von ihr intendierten Sinn eines Textes preisgeben zu können als andere Textformen. Das ist insbesondere dann der Fall, wenn Fragmente (nur) handschriftlich überliefert sind und gleichsam der Verfasser noch spürbar ist. Geführt hat das bisweilen dazu, dass das Fragment über das Werk gesetzt wurde, weil es, verknappt gesagt, dem hermeneutischen Modell des Verstehens förderlicher schien als der abgeschlossene Text, das vollendete Werk. Zumindest aber fungieren Fragmente gegenüber dem Werk als das, was Gérard Genette Epitexte genannt hat, nämlich die nicht materiell mit dem Werk (im Sinne von ,Buch') verbundenen Texte, die aber dennoch als Aufmerksamkeit und Rezeption steuernde Membranen, über die man als Rezipient in einen Text hineingelangt, fungieren können. ${ }^{17}$ Dazu rechnet Genette alle „Vortexte“, ${ }^{18}$ zu denen sich auch Fragmente zählen ließen.

Hans-Georg Gadamer hat die hermeneutische Tätigkeit in Wahrheit und Methode in einer inzwischen berühmt gewordenen Stelle als ein „Entwerfen“ von Sinn beschrieben, bei dem sich derjenige, der verstehen will, „einen Sinn des Ganzen voraus“ wirft. Aber:

Ein solcher zeigt sich wiederum nur, weil man den Text schon mit gewissen Erwartungen auf einen bestimmten Sinn hin liest. Im Ausarbeiten eines solchen Vorentwurfs, der freilich beständig von dem revidiert wird, was sich bei weiterem Eindringen in den Sinn ergibt, besteht das Verstehen dessen, was dasteht. ${ }^{19}$

Für die hier von Gadamer so eindrücklich beschriebene Methodik der Hermeneutik sind das Fragment, die Skizze, der Entwurf, kurz alle Vor-Formen späterer Werke besonders geeignet, lässt sich der am eigentlichen Werk entwickelte Sinnentwurf doch mit relativ großer Erfolgswahrscheinlichkeit dem Fragment ,vorauswerfen', auch wenn dieser Vorauswurf eigentlich ein Nach- oder noch besser Zurück-Wurf ist. Gemeint ist jene durchaus gängige Praxis der sinnverstehenden Interpretation eines Textes, die zu ihrer Absicherung auch noch das zugehörige Fragment heranzieht und aus der Übereinstimmung zwischen Fragment und zu

17 Gérard Genette, Paratexte. Das Buch vom Beiwerk des Buches, Frankfurt am Main, New York 1989, S. 354-384.

18 G. Genette, Paratexte (wie Anm. 17), S. 376-384.

19 Hans-Georg Gadamer, Hermeneutik I. Wahrheit und Methode. Grundzüge einer philosophischen Hermeneutik, 6. Aufl., Tübingen 1990, S. 271. 
deutendem ,Werk` Bekräftigung der Ausgangshypothese erfährt. Es wird also ein gewisser Grad an Kohärenz zwischen Fragment und Werk gleichermaßen behauptet wie in der Interpretation auch hergestellt bzw. herausgearbeitet.

Ausgehend von der zitierten Gadamer-Stelle hat Dieter Kafitz betont, dass derjenige, der etwas verstehen will, mit dem, was er verstehen will, in einem Zusammenhang steht, der das Verstehen erst ermöglicht. ${ }^{20}$ Auch das kann in der Logik des hermeneutischen Denkmodells neben Briefen vor allem auf dem Weg über Fragmente erreicht werden, jedenfalls dann, wenn man voraussetzt, dass diese einen unmittelbareren Blick auf den Autor zulassen als die abgeschlossenen Werke. Die Karikatur dieser Praxis wäre derjenige Literaturwissenschaftler, der den Auftrag erhält, einen Text zu interpretieren, und als erstes ins Archiv geht und nach unveröffentlichten Fragmenten zum abgeschlossenen Werk sucht. ${ }^{21}$ Man muss sich aber klarmachen, dass dabei das Wissen um das spätere Werk (im Sinne von Einzel- ebenso wie von Gesamtwerk) stets den unausgesprochenen Vergleichspunkt bildet, so dass es nicht allein das Fragment selbst ist, das als offene Form zusätzliche Bedeutung bzw. zusätzliches Wissen vermittelt. ${ }^{22}$ An seine Grenzen stößt das hermeneutische Interesse am Fragment aber da, wo Fragmente spielerisch Möglichkeiten (auch solche, die vielleicht keinen Sinn ergeben) ausprobieren, was dann eher vom Ort der Spieltheorien aus zu beschreiben wäre.

Werden die Stellen des Vorläufigen, Unvollendeten, Bruchstückhaften als Einfallstore für das Verstehen (gleichermaßen des Verfassers wie des jeweiligen Textes) konzipiert, dann ist bereits der Übergang von der traditionellen Hermeneutik zu derjenigen des Lesens angelegt, der dann zur Rezeptionsästhetik führt. Denn das Fragment bietet für das Konstrukt des impliziten, vom Text vorgegebenen idealen Lesers einerseits wichtige Leerstellen, deren Ausfüllen für ein Verständnis möglicherweise entscheidend ist, andererseits gibt es jedoch für die wenigsten literarischen Fragmente so etwas wie eine ästhetische Rezeptionsgeschichte, in der sich das poetische Potenzial eines Fragments aufgrund seiner Offenheit peu à peu in Form von Neulektüren entfalten könnte. Das aber ist eher für kanonische, vielgelesene und auch literaturwissenschaftlich vielbeachtete Texte der Fall. Für die Rezeptionsästhetik jaussscher und iserscher Provenienz

20 Vgl. Dieter Kafitz, Literaturtheorien in der textanalytischen Praxis, Würzburg 2007, S. 31.

21 In dieser Suche nach Nähe zu Autorinnen und Autoren klingt Friedrich Schleiermachers Vorschlag nach, den Sinnentwurf zu einem Werk dadurch auf die Probe zu stellen, dass man ihn mit den Lebensumständen der Autorinnen und Autoren abgleicht. Vielfach treten an diese Stelle auch Fragmente und andere Formen des Unvollendeten.

22 Nachzugehen wäre auch der Frage, ob die Fragmente Fontanes immer dann verstärkt an Interesse gewinnen, wenn die eigentlichen, geschlossenen Werke als für den Moment ,ausgeforscht gelten. 
erklärt sich daraus umgekehrt ihr Desinteresse an Fragmenten, Skizzen und Entwürfen, die kaum einmal eine Rolle spielen. Es würde allerdings auch zu einer Inflation der Bezeichnung Fragment führen, wenn man Rezeption ganz generell als einen ,Weg ins Offene“ verstände, der auf Basis von Leerstellen stets aufs Neue Fragmente hervorbringt. ${ }^{23}$

Genau umgekehrt sieht es für alle Spielarten von auch heute noch anzutreffenden Genie-Ästhetiken aus, wie sie sich vor allem in den medialen Selbstdarstellungen von Autorinnen und Autoren manifestieren. Ihnen muss die Vorstellung des in der Sekunde des Gedankens hingeschriebenen Entwurfs näherstehen als der vielleicht mehrfach überarbeitete und möglicherweise sogar von dritter oder gar vierter Hand erst lektorierte und dann gedruckte Text eines Werkes. Davon ausgehend ist schließlich noch genereller $\mathrm{zu}$ fragen, was eigentlich genau passiert, wenn Fragmente nicht nur als ,unvollendet‘, sondern als ,unvollendbar diskursiviert werden. Es findet dann eine ästhetische Verabsolutierung und geradezu ,Höchstwertung‘ einer Phase im künstlerischen und speziell literarischen Produktionsprozess statt, was wiederum impliziert, dass auch die als ,vollendet“ kodierten Werke implizit als eigentlich nicht wirklich vollendet (,vollendbar`) gedacht werden können und müssen, jedenfalls dann, wenn man sie in einen prinzipiell als unabschließbar gedachten generativen Prozess einreiht. ${ }^{24}$

\section{II.3 Generative Literaturtheorien, Literatursoziologie}

Was das Interesse am Fragment angeht, berührt sich die Genieästhetik punktuell mit den generativen Literaturtheorien, wie sie in den 1970er Jahren entwickelt und dann vielfach materialistisch und/oder literatursoziologisch erweitert

23 So hat Wolfgang Iser zur Veranschaulichung des Leerstellen-Konzepts die „Schnitttechnik“ des Fortsetzungsromans angeführt (Iser, Der Akt des Lesens. Theorie ästhetischer Wirkung. München 1976 [UTB 636], S. 296-298; Iser, Die Appellstruktur der Texte. Unbestimmtheit als Wirkungsbedingung literarischer Prosa. Konstanz 1971 [Konstanzer Universitätsreden 28], S. 17-19), seine einzelnen Teile aber eben nicht als Fragmente bezeichnet.

24 So etwa ist das Regie-Theater zu verstehen, dessen Prinzip ja darin besteht, die eigentlich längst abgeschlossenen Werke wieder neu aufzubrechen. - Als Vorläufer des Regie-Theaters lassen sich solche teleologisch-historizistischen Geistestypologien ansehen, die die Formen des Offenen (einschließlich des Fragments) mal mehr mal weniger explizit als repräsentative Symbole von ,Unendlichkeit“ gedeutet haben. Das Spektrum reicht hier von Fritz Strich, Deutsche Klassik und Romantik, oder Vollendung und Unendlichkeit. Ein Vergleich. München 1922, bis hin zu Volker Klotz, Offene und geschlossene Form im Drama. München 1960. - Für diese Hinweise danke ich Jürgen Link. 
wurden, wie beispielsweise in dem 1980 veröffentlichten Literatursoziologischen Propädeutikum von Jürgen Link und Ursula Link-Heer. ${ }^{25}$ Ziel dieser generativen Literaturtheorien war und ist es - darin an die russischen Formalisten anschließend -, möglichst den gesamten Apparat an Regeln zu beschreiben, nach denen ein Text geschrieben ist, was es zugleich ermöglicht, aus diesem Set an Regeln ähnliche Texte $\mathrm{zu}$ produzieren.

Das Fragment bietet für die generative Literaturtheorie dabei zwei Möglichkeiten: erstens durch den Vergleich mit wirklich zu Ende geschriebenen Texten desselben Autors die besonders gute Chance, die generativen Regeln des Schreibens kontrastiv sichtbar zu machen; zweitens durch Aufzeigen der Unterschiede im Regelapparat von ,fertigen' Werken und ,fragmentarischen' Texten ästhetische Entscheidungen deutlich zu machen und von ihnen ausgehend Text-Charakteristika zu bestimmen. Das Fragment wäre dann so etwas wie das vergleichbare Andere im Eigenen. Da, wo es keine Fragmente gibt, werden von den generativ orientierten literaturtheoretischen Ansätzen gern auch Texte zum Vergleich geeigneter Autorinnen und Autoren aus der gleichen Zeit, aus ähnlicher sozialer Stratifikation und möglichst auch ähnlicher Gattung herangezogen. Dieser generativ denkende Zugriff auf Fragmente ist sicherlich eine der verbreitetsten Formen der analytischen und interpretatorischen Verwendung von Fragmenten (auch in Kombination mit anderen Literaturtheorien als der generativen), auch wenn dieses vielfach praktizierte Vorgehen eher selten in direktem Rekurs auf das literaturtheoretische Paradigma der generativen Literaturtheorie reflektiert wird.

\section{II.4 Strukturalismus, Diskursanalyse}

Schauen wir uns weiter Strukturalismus und Diskursanalyse an. Für den klassischen Strukturalismus, der die Sinnangebote von Texturen in erster Linie aus ihrer Zeichenhaftigkeit zu entwickeln sucht, macht der Überlieferungsstatus bzw. die Nähe zu dem, was emphatisch als abgeschlossenes Werk angesehen wird, kaum einen Unterschied. Von daher ist das Fragment für ihn zunächst einmal eine Textur neben zahlreichen anderen. Auch in diachroner Perspektive ändert sich das nicht wesentlich, geht es doch in ihr darum, die herausgearbeiteten grundlegenden semantischen Strukturen eines Textes für verschiedene synchrone Schnitte oder auch für verschiedene Autorinnen und Autoren zu verglei-

25 Jürgen Link/Ursula Link-Heer, Literatursoziologisches Propädeutikum. Mit Ergebnissen einer Bochumer Lehr- und Forschergruppe Literatursoziologie 1974-1976 (Hans Günther, Horst Hayer, Ursula Heer, Burkhardt Lindner, Jürgen Link), München 1980 (UTB 799). 
chen. Denkbar wäre es, strukturale Vergleiche auch für verschiedene Gattungen zu unternehmen, doch scheinen solche Versuche, bei denen das Fragment dann wenn auch nicht als Gattung, so doch vielleicht als Genre oder Subgenre der ,Formen des Offenen' angesehen werden könnte, bisher nicht unternommen worden zu sein. Möglicherweise wäre das auch nicht sehr ertragreich.

Indem sich die Diskursanalyse für quer durch ganze Bündel von Texturen unterschiedlichster Provenienz wiederkehrende Elemente der Rede interessiert, behandelt sie Fragmente und ausgearbeitete Werke gleich. Differenzierungen kommen dabei dadurch ins Spiel, dass in unterschiedliche diskursive Positionen (die kohärente wertende Benutzung ganzer Cluster von Diskurselementen) unterschieden wird. Fragmente ließen sich dann von abgeschlossenen Werken dadurch unterscheiden, dass die zwischen beiden liegende Entwicklung als Modifikation der ursprünglichen Diskursposition verstanden oder eben ihre Kontinuität konstatiert wird. In der spezifischen Form der Interdiskurstheorie fragen diskursanalytische Ansätze zudem nach denjenigen analogiebildenden Elementen von Texten, die Brücken der Allgemeinverständlichkeit zwischen Spezialdiskursen schlagen, insbesondere nach Metaphern und Symbolen. Fragmente können dabei insofern interessant sein, als sie möglicherweise in besonders starkem Maße auf Bildlichkeiten zurückgreifen und ebenso verstärkt verschiedene gesellschaftliche Teilbereiche und ihre Diskurse aufeinander beziehen. Um solche Brückenschläge ging es letztlich auch der romantischen Fragment-Praxis, wobei vor allem Philologie, Philosophie, Poetik, Kritik und Geschichte zu einer Art von universalem Diskurs integriert werden sollten.

\section{II.5 Psychoanalytische Literaturwissenschaft, Dekonstruktion}

Bleiben noch psychoanalytische Literaturwissenschaft und Dekonstruktion. Eine an Sigmund Freud orientierte psychoanalytische Literaturwissenschaft kann das Fragment gegenüber dem Werk als eine verschüttete, vom Werk her nicht mehr unmittelbar zugängliche Textstufe und darüber hinaus der Tendenz nach auch als eine solche Autoräußerung verstehen, die es analog zur psychoanalytischen Sitzung ,wieder zu holen' gilt. ${ }^{26}$

26 Eine Rolle spielt das Fragment auch in der für die Literaturwissenschaft relevant gewordenen Träume-Theorie, die das Fragment als denjenigen Bestandteil persönlicher Erfahrung versteht, der sich nicht identitätsstiftend integrieren lässt. Zu denken ist hier etwa an das Konzept der ,postmemory', das davon ausgeht, dass bestimmte Erinnerungen (innerfamiliär) wirken, ohne den ,Nachgeborenen` erinnerbar zu sein. Sie markieren also Leerstellen, die auf spätere Bewusst- 
Für dekonstruktivistische Literaturtheorien, die jeden Text, ob abgeschlossenes Werk oder Bruchstück, als Fragment verstehen, stellen Fragmente - zugespitzt formuliert - prekärerweise stets schon das Ziel der eigenen Bemühungen dar. So heißt es bei Jacques Derrida in Die Schrift und die Differenz: „Das Fragment ist kein bestimmter Stil und kein bestimmtes Scheitern, es ist die Form des Geschriebenen.“27 Denn in der Konfrontation und im Vergleich mit den abgeschlossenen Werken lassen sich Fragmente auch als Formen der Dekonstruktion, des Offenen verstehen, als Elemente jener ,Spur‘, die das dekonstruktivistische Bemühen einer Poetologie der Möglichkeiten allererst offenlegen will, zielt die Dekonstruktion doch darauf ab, nach Widersprüchen und Differenzen zwischen dem offensichtlich Gemeinten und dem tatsächlich (auch noch) Gesagten zu fragen. Die verschiedenen Spielarten der Dekonstruktion greifen daher mit Vorliebe mehrdeutige Zeichenkomplexe auf, wie Metaphern, Metonymien und Symbole; Zeichen werden durch eingefügte Bindestriche neue Bedeutungen gegeben (z. B. ,Wiederholung' und ,Wieder-Holung'). Hier können Fragmente Ansatzpunkte bieten, allerdings würde das Fragment dann immer schon ein Stück weit die Arbeit der Dekonstruktion leisten, die als Ausgangspunkt jedoch selbst der „Geschlossenheit des Textes sowie seiner [...] Identifizierbarkeit und definite[n] Abgrenzbarkeit gegenüber [...] Kon-Texten“ bedarf. ${ }^{28}$ Zudem hätte man es mit einer Umkehr der Blickrichtung zu tun, wenn nicht mehr die abgeschlossenen Werke dekonstruiert werden, sondern die Fragmente.

Stellt sich der Weg vom Fragment im Sinne von ,Entwurf‘ zum ,Werk‘ als einer der Vervollständigung oder auch Vervollkommnung dar, so liefe die Blickrichtung der Dekonstruktion und der psychoanalytischen Literaturwissenschaft jeweils genau in die umgekehrte Richtung; bei der Dekonstruktion mit dem Ziel, eindeutige Sinnbildungen in der Schwebe und damit für vielfältige Sinnmöglichkeiten offen zu halten.

machung drängen. Für die Literatur ist dies insofern relevant, als fiktional-narrative Konstrukte um das fragmentarisch Weiterwirkende geschaffen werden. Vgl. dazu Marianne Hirsch, The Generation of Postmemory: Writing and Visual Culture After the Holocaust, New York 2012.

27 Jacques Derrida, Die Schrift und die Differenz, Frankfurt am Main 1976 (suhrkamp taschenbuch wissenschaft 177), S. 111.

28 P. Strohschneider, Fragment ${ }_{2}$ (wie Anm. 6), S. 625. 


\section{II.6 Kurzes Zwischenfazit}

Was der - zugegeben - sehr knappe und vielfach verkürzend darstellende Parforceritt durch das Spektrum der gängigen Literaturtheorien für den Umgang mit Fragmenten gezeigt hat, ist vor allem, dass es zu erstaunlichen Allianzen und Überlagerungen kommt, dass solche theoretischen Paradigmen, die man gewohnt ist als in starker Opposition zueinander stehend zu sehen, mit Blick auf die Logik ihres Umgangs mit Fragmenten plötzlich nahe zusammenrücken.

Lässt sich dieser Befund auch für die tatsächliche Praxis der literaturwissenschaftlichen Beschäftigung mit den Fragmenten Fontanes aufrechterhalten? ${ }^{29}$ Dazu soll auf einige wenige Beispiele eingegangen werden, weist die FontaneBibliographie jenseits des Romanfragments Mathilde Möhring doch nur rund zwei Dutzend sich dezidiert mit den Fragmenten beschäftigende Arbeiten aus. ${ }^{30}$

\section{Die tatsächliche Praxis der Beschäftigung mit Theodor Fontanes Fragmenten}

Ein in seiner Komplexität erstaunliches Beispiel für die (unreflektiert bleibende) Überlagerung ganz unterschiedlicher theoretischer Paradigmen bietet bereits Hermann Frickes 1938 erschienene Studie Theodor Fontanes letzter Romanentwurf. Die Likedeeler, ${ }^{31}$ deren Rahmenerzählung den Störtebecker-Stoff mit der gesamten Spanne des fontaneschen Lebens korreliert, Autor und Stoff also auf das Engste zusammenbringt, womit sich Fricke zunächst einmal dem schleiermacherschen Modell von Hermeneutik anschließt, nämlich den Sinnentwurf zu einem Werk dadurch auf die Probe zu stellen, dass man ihn mit den Lebensumständen des Autors abgleicht. Fricke betreibt das so weitgehend, dass er Fontanes Leben von der frühesten Jugend und den Störtebecker-Spielen in Swinemünde an

29 Einen Überblick nach Arbeitsweisen, Stoffen, Themen und Motiven sowie der Überlieferung von Fontanes Fragmenten selbst bieten Bettina Plett, Fragmente und Entwürfe. In: FontaneHandbuch, hg. von Christian Grawe und Helmuth Nürnberger, Stuttgart 2000, S. 693-705, sowie Christine Hehle/Hanna Delf von Wolzogen, Einleitung. In: Theodor Fontane, Fragmente. Erzählungen, Impressionen, Essays, im Auftrag des Theodor-Fontane-Archivs hg. von Ch. Hehle und H. Delf von Wolzogen, Bd. I: Texte, Berlin, Boston 2016, S. XI-XXXIV.

30 Wolfgang Rasch, Theodor-Fontane-Bibliographie. Werk und Forschung, 3 Bde., in Verbindung mit der Humboldt-Universität zu Berlin und dem Theodor-Fontane-Archiv hg. von Ernst Osterkamp und Hanna Delf von Wolzogen, Berlin, New York 2006, S. 2035-2040.

31 Hermann Fricke, Theodor Fontanes letzter Romanentwurf. Die Likedeeler, Rathenow 1938. 
bis zu seinen letzten Tagen in die verschiedensten Analogierelationen zum Störtebecker-Stoff und seinen diversen Formen der Behandlung bei Fontane stellt, und zwar vom Balladenentwurf bis hin zum „unvollendet gebliebenen großen historischen Roman ,Die Likedeeler““.32

Dieses letzte Zitat macht deutlich, dass Fricke den Abgleich von Biographie und Text aber zugleich auch in eine teleologische Aufwärtslinie des „dichterischen Schöpfertums“33 Fontanes einbindet, was ihn zwingt, die Bezeichnungen ,Fragment‘ und ,Entwurf‘ möglichst zu vermeiden. Diese Linie konzipiert Fricke als eine Art autorbezogene geistesgeschichtliche Entwicklung aufwärts, die dem Nachweis dienen soll, dass Fontanes Alterswerk den Höhepunkt einer lebenslangen Entwicklung darstellt und keinesfalls einen Einbruch. Das wiederum wird vor allem dadurch erreicht, dass das „Likedeeler“-Fragment auf nahezu alle ,vollendeten' Werke Fontanes bezogen wird, mit dem Effekt der Konstitution eines integralen Gesamtwerkes vom einzelnen Fragment aus.

Heute fast schon modern anmutend wird dieser Mix aus hermeneutischem und geistesgeschichtlichem Denken durch längere Passagen zu geschichtlichen und ansatzweise sozialgeschichtlichen Informationen $\mathrm{zu}$ den Vitalienbrüdern sowie durch die Behandlung des Stoffes bei anderen Autoren ergänzt. Es folgt ein kommentierendes, chronologisches Close Reading der „Likedeeler“-Fragmente, bei dem die unausgesprochenen und wahrscheinlich auch unreflektierten literaturtheoretischen Verortungen bisweilen durchscheinen, so etwa das geistesgeschichtliche Denkmodell der Übereinstimmung von Gestalt und Geist, wenn es heißt: „So stark auch der geschichtliche Hintergrund bei dem Entwurf wirken mochte, entscheidend blieb für den Dichter die dichterische Formgebung. Ihr Ziel geht aus einer ergänzenden Aufzeichnung hervor.“34

Mit all dem ist Fricke letztlich bemüht, durch Zusammenführung aller zum Störtebecker-Stoff bei Fontane vorfindlichen Fragmente, Notizen, Entwürfe, Bemerkungen in Briefen, Quellen und biographischen Informationen jenen großen „Likedeeler“-Roman zu konstituieren, den Fontane gar nicht geschrieben hat. Der Status der Fragmente als Formen des Offenen wird damit insgesamt geradezu konterkariert, was die gleichsam Tatsachen schaffende Überschrift des Schlusskapitels mehr als deutlich macht: „Bedeutung des Likedeeler-Romans“. ${ }^{35}$ Darin heißt es, den Clash der literaturtheoretischen Leitideen noch einmal deutlich machend:

32 H. Fricke (wie Anm. 31), S. 4.

33 H. Fricke (wie Anm. 31), S. 4.

34 H. Fricke (wie Anm. 31), S. 42.

35 H. Fricke (wie Anm. 31), S. 157. 


\begin{abstract}
Überblicken wir Fontanes Altersschaffen seit seiner letzten schweren Erkrankung im Jahre 1891, so sehen wir neben den großen autobiographischen Schriften, den märkischen Romanen und den Bemühungen um das Ländchen Friesack als eine völlig einzigartige Erscheinung die Arbeit an den „Likedeelern“. Sie steht im Gegensatz zu Fontanes Romanschaffen von 1884 bis 1894 und im Gegensatz zu seinem letzten vollendeten Roman. Sie knüpft allerdings nur zur Hälfte an seine Balladen und balladesken Novellen an und sucht eine höhere Einheit zwischen ihnen und seinen Berliner Romanen. Wie Hebbel einst das Novantike als Ideal seines Dramas anstrebte, so sollten die Likedeeler den deutschen Roman auf eine ganz neue Stufe führen, eine innere Brücke zwischen Romantik und Realismus bilden. Diese innerlich gewandelte Form des historischen Romans sollte das Mittel werden, um der Begründung einer neuen Epoche des deutschen Romans zu dienen. ${ }^{36}$
\end{abstract}

Integration des Gesamtwerkes, Vermittlung von Romantik und Realismus plus Neubegründung des deutschen Romans, das ist nicht wenig, was einem Fragment hier an fragmentarischer Phantasie der Rezeption aufgebürdet wird.

Vergleicht man Frickes Arbeit mit Julius Petersens knapp zehn Jahre zuvor erschienener Studie zu „Allerlei Glück“ als - so der Titel - „Fontanes erste[m] Berliner Gesellschaftsroman “, ${ }^{37}$ dann zeigt sich ein anderes Bild, und zwar das einer Arbeit, die den Fragment-Charakter des Textes sowohl philologisch wie auch konzeptionell durchaus ernst nimmt, nämlich als „Versuch eines Berliner Zeit- und Gesellschaftsromans“, als „gewissermaßen die Urzelle, aus der sich neue Artungen und Lebewesen abspalten sollten“:

Er stellt das Schulstück einer neuen Gattung dar, das mit den späteren Berliner Erzählungen in Motiven, in Charaktertypen und Mitteln der Erzähltechnik als Vorstudie eng verbunden bleibt. Diese Beziehungen geben dem unausgeführten Werk eine besondere entwicklungsgeschichtliche Bedeutung [...]. ${ }^{38}$

Zurückgebunden an die unternommene kleine Revue literaturtheoretischer Logiken des Fragments ließe sich sagen, dass sich Petersen auf Basis einer im besten Sinne positivistischen Dokumentation und Sichtung der vorhandenen fontaneschen Text-Materialien in Richtung einer struktural-generativen Literaturtheorie bewegt, die nach der Entwicklung von Charakteren und Motiven fragt, die teilweise sozialgeschichtlich kontextualisiert werden (etwa wenn die Berliner Adressbücher hinsichtlich der Namen der Figuren, ihrer Wohnorte und

36 H. Fricke (wie Anm. 31), S. 140.

37 Julius Petersen, Fontanes erster Berliner Gesellschaftsroman. Sonderausgabe aus den Sitzungsberichten der Preußischen Akademie der Wissenschaften phil.-hist. Klasse 1929, XXIV, Berlin 1929.

38 J. Petersen (wie Anm. 37), S. 5. 
ihrer sozialen Stellung ausgewertet werden), dabei aber nicht wie Fricke der Versuchung erliegt, die Fragmente dominant über alles andere zu setzen. Vielmehr denkt Petersen von den Fragmenten ausgehend in möglichen generativen Szenarien:

Wenn der Roman nach diesem Plan durchgeführt worden wäre, so hätte [...] die „Lebensund Sittenschilderung“ gegenüber dem „Grundstock“ das Übergewicht behalten. Der Grundstock enthielt die dreifachen Elemente eines Ideen- und Bildungsromanes, eines humoristischen Familienromanes und eines sozialen Romanes. ${ }^{39}$

Ich überspringe Walter Müller-Seidels Überlegungen zu „Allerlei Glück, “40 weil es um eine übergreifende literaturgeschichtliche Reflexion zur Darstellung von Glück im 18. und 19. Jahrhundert geht, für die das Fragment Fontanes nur den äußeren Anlass darstellt, aber nicht anders als die Romane behandelt wird, nämlich als Dokument zum Glücks-Verständnis in der Literatur nicht nur des 19. Jahrhunderts.

Weiter geht da Hans-Heinrich Reuters zuerst 1966 veröffentlichte Analyse zu Fontanes Prosafragment „Koegels-Hof Nummer drei“ ${ }^{41}$ denn Reuter ist einerseits bemüht, das Fragment auf weitere Texte des Offenen bei Fontane zu beziehen und auf diese Weise „mit seinem Alterswerk schlechthin“ zu verbinden, nimmt andererseits aber auch „die Spezifik des Fragments von 1880 in den Blick“, um den „Ort sichtbar“ zu machen, „,den es in diesem Werk einnimmt“. ${ }^{42}$ Die Verankerung im ,Fragment-Gesamtwerk ${ }^{\star}$ erfolgt dabei ebenso wie das Herausstellen der Besonderheit durch Struktur- und Themenvergleich: Die Art der schnellen Niederschrift der Entwürfe bleibe gleich, spezifisch seien dagegen die beiden Teilthemen des ökonomischen Rückschlags und der „Deklassierung des selbständigen Handwerkerstandes". ${ }^{43}$

Noch ernster nimmt Peter Wruck in seiner Analyse zu Fontanes „Die preußische Idee“ ${ }^{\star 44}$ von 1982 den Entwurfscharakter des zu analysierenden Textes und

39 J. Petersen (wie Anm. 37), S. 37.

40 Walter Müller-Seidel, „Allerlei Glück“. Über einen Schlüsselbegriff im Romanwerk Theodor Fontanes. In: Zeitwende, 48, 1977, S. 1-17.

41 Hans-Heinrich Reuter, Theodor Fontane, „Koegels Hof Nummer drei“. Fragment einer ungedruckten Erzählung. In: H.-H. Reuter, Dichters Lande im Reich der Geschichte. Aufsätze zur deutschen Literatur des 18. und 19. Jahrhunderts, Berlin, Weimar 1983, S. 289-307. Der Aufsatz erschien zuerst in Sinn und Form, 1, 1966, S. 1131-1152.

42 H.-H. Reuter (wie Anm. 41), S. 299.

43 H.-H. Reuter (wie Anm. 41), S. 300.

44 Peter Wruck, Fontanes Entwurf „Die preußische Idee“. In: Fontane Blätter, 34, 1982, S. 169190. 
entwickelt - ausgehend von einer genauen philologischen Analyse der beiden Teile des Fragments - eine Lesart auf Basis sozialgeschichtlicher Kontextualisierungen, die sich als an das Theorieparadigma der Sozialgeschichte der Literatur angelehnt verstehen lässt, bei ergänzenden Passagen, die tendenziell ideengeschichtlich orientiert sind.

Bleibt zuletzt noch, auf einen Beitrag einzugehen, der einen vielfach als abgeschlossen rezipierten Text Fontanes gleichsam zurück in die Fragmentarität holt, nämlich Gabriele Radeckes Nachweis der vielfältigen „Spuren der Unvollendetheit“ in Mathilde Möhring von 2003. ${ }^{45}$ Das leitende Forschungsparadigma ist hier das einer sehr genauen Editionsphilologie, insbesondere Manuskriptanalyse, erweitert um das lediglich punktuelle Heranziehen von Informationen biographischer Art und bei Verzicht auf weitergehende Interpretationen, wodurch dann auch keine weiteren theoretischen Optionen ins Spiel kommen. Allerdings wäre ebenso wie das Spektrum des Zusammenspiels von Literaturtheorien und Fragment auch dasjenige von Fragment und theoretischen Konzeptionen des Edierens genauer $\mathrm{zu}$ sichten. ${ }^{46}$

\section{Fazit}

Ausgangspunkt war, die Formen des Offenen und einige ausgewählte literaturtheoretische Positionen aufeinander zu beziehen und danach $\mathrm{zu}$ fragen, welcher Status Fragmenten, Entwürfen, Notizen und anderen Formen des Offenen aus Sicht der verschiedenen Literaturtheorien eigentlich zukommt, um dann nach den meist unausgesprochen bleibenden theoretischen Optionen der FragmentForschung zu Fontane zu fragen. Gezeigt hat sich, dass es keine ,lupenreinen“ Theorieorientierungen gibt, sondern in der Regel verschiedene Paradigmen miteinander verknüpft werden, die sich überlagern. Dabei kommen theoretische Ansätze eng zueinander zu stehen, von denen man zunächst einmal annehmen würde, dass sie einander diametral entgegengesetzt wären. Weiter ist deutlich

45 Gabriele Radecke, „Leider ,nicht“ druckfertig“. Spuren der Unvollendetheit in Theodor Fontanes „Mathilde Möhring“. In: Schrift - Text - Edition. Hans Walter Gabler zum 65. Geburtstag, hg. von Christiane Henkes u. a., Tübingen 2003, S. 221-230.

46 Vgl. dazu Rüdiger Nutt-Kofoth (Konzepte der Fragmentedition und ihre Probleme. In: Fragment und Gesamtwerk. Relationsbestimmungen in Edition und Interpretation, hg. von Matthias Berning, Stephanie Jordans und Hans Kruschwitz, Kassel 2015, S. 13-27), der nach dem Zusammenhang zwischen den jeweiligen Modi des editorischen Umgangs mit Fragmenten, den (unausgesprochenen) Prämissen der Editoren und deren Erkenntnisinteressen fragt. 
geworden, dass zwischen Fragmentarität auf den Ebenen der Produktion, der Produkte und der Rezeption deutlich zu unterscheiden ist.

Da das meist eher pragmatisch geschieht und nicht auf einer Metaebene reflektiert wird, sei noch einmal wiederholt: Der Bezug von literaturwissenschaftlicher Arbeit an Fragmenten - sei es interpretatorische, sei es editorische - auf Literaturtheorien kann uns die eine oder andere Voraussetzung, Prämisse und Bedingung unseres Bemühens deutlicher machen, als sie uns in der praktischen literaturwissenschaftlichen Arbeit vor Augen steht. 


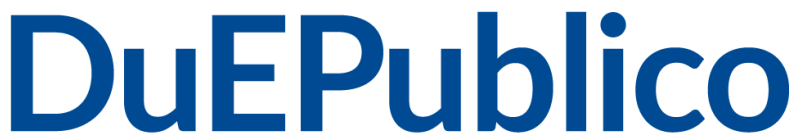

Duisburg-Essen Publications online
UNIVERSITÄT

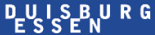

Offen im Denken

$\mathbf{U b} \mid \begin{aligned} & \text { universitäts } \\ & \text { bibliothek }\end{aligned}$

Dieser Text wird über DuEPublico, dem Dokumenten- und Publikationsserver der Universität Duisburg-Essen, zur Verfügung gestellt. Die hier veröffentlichte Version der EPublikation kann von einer eventuell ebenfalls veröffentlichten Verlagsversion abweichen.

DOI: $\quad 10.1515 / 9783110539493-001$

URN： urn:nbn:de:hbz:464-20210224-125106-1

Parr, Rolf: Eine kleine Revue literaturtheoretischer Logiken des Fragments.

In: Formen ins Offene. Zur Produktivität des Unvollendeten / Delf von Wolzogen, Hanna ; Hehle, Christine (Hrsg.). Berlin ; Boston: De Gruyter, 2018. - e-ISBN (PDF)

978-3-11-053949-3. DOI: https://doi.org/10.1515/9783110539493, S. 3-20

(C) 2018 Walter de Gruyter GmbH, Berlin/Boston. Alle Rechte vorbehalten. 